\title{
Pearls in autoimmunity
}

\author{
Jozélio Freire de Carvalho • \\ Rosa Maria Rodrigues Pereira • \\ Yehuda Shoenfeld
}

Received: 2 January 2011/Accepted: 11 January 2011/Published online: 23 March 2011

(C) Springer-Verlag 2011

\begin{abstract}
This manuscript does a review of the more frequent issues published at Autoimmunity Reviews, Journal of Autoimmunity and Autoimmunity in the period of JanuaryDecember 2009. The following topics were commented: (1) multiple sclerosis (MS) and its relationships with Epstein Barr infection, with vitamin D polymorphism and the new modalities of MS treatment. (2) Type 1 diabetes and genetic discovers, studies with GAD 65 and IA- 2 autoantigen and the association T1D and autoimmune organ-specific diseases. (3) Autoimmune thyroid disorders and its association with susceptibility genes and polymorphisms. (4) Multiplex autoantibody profiling approaches in MS and rheumatoid arthritis. (5) Th17 cytokine in primary biliary cirrhosis, experimental autoimmune encephalomyelitis and celiac disease. (6) Vitamin D and experimental autoimmune prostatitis and pulmonary alveolar proteinosis.
\end{abstract}

Keywords Autoimmunity · Multiple sclerosis .

Diabetes · Thyroid disorders · Vitamin D .

Autoantibodies

J. F. de Carvalho $(\bowtie) \cdot$ R. M. R. Pereira

Rheumatology Division, Disciplina de Reumatologia, Faculdade de Medicina, Universidade de São Paulo, Av. Dr. Arnaldo 455, $3^{\circ}$ andar, Sala 3105, São Paulo, SP 01246-903, Brazil

e-mail: jotafc@gmail.com

Y. Shoenfeld

Department of Medicine B, Center for Autoimmune Diseases, Sackler Faculty of Medicine, Sheba Medical Center, Tel-Aviv University, Tel-Hashomer, Israel

Y. Shoenfeld

Incumbent of the Laura Schwarz-Kipp Chair for Research of Autoimmune Diseases, Tel-Aviv University, Tel-Aviv, Israel

\section{Introduction}

PubMed searches include only the following periodics: Autoimmunity Reviews, Journal of Autoimmunity and Autoimmunity, this manuscript did a review of the more frequent issues published in the period of JanuaryDecember 2009.

The following topics will be herein summarized: (a) multiple sclerosis (MS) and its relationships with Epstein-Barr infection, with vitamin D polymorphism and the new modalities of MS treatment. (b) Type 1 diabetes (T1D) and genetic discovers, studies with GAD 65 and IA2 autoantigen and the association T1D and autoimmune organ-specific diseases. (c) Autoimmune thyroid disorders and its association with susceptibility genes and polymorphisms. (d) Multiplex autoantibody profiling approaches in MS and rheumatoid arthritis. (e) Th17 cytokine in primary biliary cirrhosis, experimental autoimmune encephalomyelitis and celiac disease. (f) Vitamin D and experimental autoimmune prostatitis and pulmonary alveolar proteinosis.

\section{Multiple sclerosis}

The literature brings the idea that vaccination against Epstein-Barr and also antiviral treatment of this condition could inhibit the progression or else cure multiple sclerosis (MS) originates from the fact of that more than $99.5 \%$ of the patients with MS are positive for Epstein-Barr virus (EBV) serology as compared to $94.0 \%$ of controls [1]. Pender proposes an autoimmune hypothesis that EBVinfected autoreactive B cells attack the target organ where they produce pathogenic autoantibodies and provide costimulatory survival signals to autoreactive $\mathrm{T}$ cells, in genetically susceptible people [2,3]. In agreement with this 
pathophysiology, Fraussen et al. [4] demonstrated that autoreactive B lymphocytes are detected in MS cerebrospinal fluid, and these cells are responsible for the immunoglobulin production and oligoclonal band formation seen in this neurological disorder, in comparison with healthy people where these B cells are not observed.

Another mechanism associated with MS pathophysiology is the vitamin D status. In this line, polymorphisms of the active metabolite vitamin D receptor (VDR) have major effects on vitamin D function and its metabolism, as well as on immune regulation. Indeed, in a recent study, the authors concluded that the VDR plays a role in the regulation of vitamin D metabolism in MS patients and in healthy controls, and that the Fok-I polymorphism affects this regulation [5]. Furthermore, other studies show a significant difference of genotype distribution frequency between the MS patients and controls for the functional exon 9 VDR marker Taq $I$ and for the Apa I alleles [6].

Regarding treatment, as the available immunomodulatory therapies for MS patients are only slightly effective and do not avoid the progression of neurological deficits, Payne et al. [7] reviewed the possibility of using new immunomodulators that act on degraded myelin growth inhibitory molecules and neuroregenerative therapies (e.g. stem cell therapy), or the combination of these modalities. These experimental treatments appear exciting, although much labor is still needed to make certain their long term safety and efficacy.

\section{Type 1 diabetes mellitus}

Type 1 diabetes (T1D) is a genetically conditioned autoimmune disease in which several polymorphisms genes have been described. Recently, Settin et al. [8] studying cytokine-related polymorphisms demonstrated association to TNF- $\alpha$ and IL-1Ra genes among Egyptians diabetic patients and suggest that these polymorphism could be considered as genetic markers for T1D.

Another interesting genetic study showed that the PTPN22 polymorphism is associated to T1D exclusively in children exposed to cow's milk during early infancy (before the age of 6 months) suggesting a relationship between genetic and environmental factors [9]. A Japanese study demonstrated that a FOXP3/Scrufin polymorphism was associated with adult onset T1D, especially in females, and slowly progressive T1D. This gene alteration could be implicated with a regulatory $\mathrm{T}$ cells dysfunction [10].

HLA-class II genes are also implicated in susceptibility for human T1D. Elagin et al. [11] demonstrated in an animal model in the context of human HLA-class II diabetes-susceptibility genes, that immunization with a human autoantigen (GAD65) results in the development of a severe insulitis and low levels of autoantibodies. These authors bring a new animal model which helps studies on GAD65 autoimmunity in the scenario of HLA-DR8.

Most T1D mellitus is caused by autoimmune pancreatic beta-cell destruction and various antigens are implicated in this process such as, glutamic acid decarboxylase-65 (GAD65), islet cell antigen (IA-2), insulin, and isletspecific glucose-6-phosphatase catalytic subunit related protein. Investigating the role of the GAD65, Kanazawa et al. [12] using GAD65 knockout non-obese diabetes (NOD) splenocytes and transferring to NOD-SCID system, the authors observed that the onset of diabetes in recipients of knockout splenocytes was significantly delayed as compared to recipients of wild-type splenocytes. This study suggested that anti-whole GAD65-reactive T cells have the ability to regulate the development of T1D.

The role of GAD65 was recently evaluated using a hydroxyl radical modified antigen. The ROS oxidation of GAD65 caused conformational perturbation, generating highly immunogenic neoepitopes. The authors concluded that this neoantigen could be one of the factors to induce T1D autoantibodies [13].

Another work studying the epitopes of the IA-2 autoantigen, the authors identified a region of IA-2 (831-860) frequently recognized by antibodies in T1D and demonstrates that these responses stimulate $\mathrm{T}$ cells to produce interleukin-10 [14].

Type 1 diabetes could be associated with other autoimmune disorders such as thyroid disease, autoimmune gastritis, celiac disease and Addison's disease. Tsirogianni et al. [15] suggested screening organ-specific autoantibodies in diabetic patients in order to detect early development of these associated autoimmune diseases.

Three additional articles concerning this field were also published at Journal of Autoimmunity [9, 11, 14].

\section{Thyroid disorders}

Various susceptibility genes, including HLA, for autoimmune thyroid diseases have been identified. HLA-DR3 has been described strongly associated with Graves' disease (GD) and the frequency of this HLA was generally 40-55\% in GD patients, compared to $15-30 \%$ in the general population, resulting in a relative risk for people with $H L A-D R 3$ of 3-4 and recent studies have suggested that the primary susceptibility allele in GD is indeed HLA-DR3 $\left(H L A-D R B 1^{*} 03\right)$ [16]. Very recently, an arginine substitution at position 74 of the DR $\beta 1$ chain of HLA-DR3 (DRb1-Arg74) has been described to be associated with GD and also related to thyroglobulin variants. This amino acid substitution originates a significant deforming in the peptide binding pocket of HLA-DR, affecting the binding 
of pathogenic thyroid peptides. This knowledge brings new ideas regarding treatment using antagonist agents or peptide to HLA-DR modulation [17]. On the other hand, non-HLA genes including the CTLA-4, CD40, PTPN22, thyroglobulin, and TSH receptor genes has been also implicated in autoimmune thyroid disease [18].

Interleukin-23 receptor single nucleotide polymorphisms has been related with Graves' oftalmopathy (GO). In a case control study with North American Caucasian subjects, the A allele of rs2201841 was demonstrated to be strongly associated with GO [19] although this genetic alteration was not observed in Japanese individuals [20].

Regarding clinical studies, our group have demonstrated for the first time in juvenile onset of systemic lupus erythematosus that thyroid disorders are associated with a mild lupus presentation in comparison to those patients without this endocrinological disease [21].

\section{Multiplex autoantibody profiling approaches}

Multiplex arrays are one of the technologies which are able to detect autoantibodies by measuring simultaneously thousands of markers from a unique biological sample. The main rationale of diagnostic tests is making an early and accurate diagnosis, however it increases the probability of false positive and false negative results.

Application of this technology has been recently used in MS using a autoantibody profiling with promising results regarding the generation of a so-called MS-specific autoantibody fingerprint. Somers et al. [22] applied this technique for MS cerebrospinal fluid identified a new panel of eight antigenic targets with $45 \%$ sensitivity and $86 \%$ specificity for this neurological autoimmune disease.

Since antibodies directed to citrullinated peptides (antiCCP) showed a similar sensitivity but a more elevated specificity than the rheumatoid factor (RF), a multiplex approach to detect RF in combination with ELISA antiCCP test was recently evaluated in patients with rheumatoid arthritis to increase the sensitivity of RF. The authors could observe in a prospective study that multiplex cytofluorimetric test appeared to be more sensitive and specific than nephelometric assay for RF detection [23].

\section{Interleukin-17-secreting $T$ cells (Th17)}

The emerging role of interleukin-17 as an important proinflammatory cytokine, produced primarily by a new $\mathrm{T}$ helper cell subset termed Th17, has received significant interest in the pathogenesis of several human autoimmune diseases and animal models of autoimmunity. Differentiation of Th17 cells is driven by the simultaneous presence of transforming growth factor-beta (TGF-beta) and some inflammatory cytokines (IL-6, IL-21) [24].

The Th17 role in the pathophysiology of primary biliary cirrhosis (PBC) was demonstrated in a recent study using human and murine model of this disease. The authors found an increase in the frequency of positive IL-17 T lymphocytes in liver tissues from PBC patients, and also increased IL-17 production in mice cocultures of splenic T cells and in liver non-parenchymal cells, suggesting a role of the Th17 in liver autoimmunity [25].

This cytokine was also studied in experimental autoimmune encephalomyelitis (EAE). The authors evaluated the role of thrombospondin-1 (TSP-1), a protein that could be implicated in the production of IL-17 through TGF-beta activation of TGF-beta, on the generation of an encephalitic Th17 response in EAE. This study demonstrated that TSP-1 participates in the differentiation of Th17 cells through its ability to activate latent TGF-beta, and enhances the inflammatory response in EAE [26].

This Th17 cytokine has also been studied in intestinal biopsies from celiac disease patients with active disease, remission disease and those after in vitro gliadin exposition. Potent Th17 responses were observed in active disease, whereas short incubation with gliadin of biopsies showed a tendency of upregulation of Th17, suggesting a importance of Th17 in active phase of celiac disease [27].

\section{Vitamin D and autoimmunity}

Vitamin D has effects in modulating the immune response including inhibitory effects on T cells, B cells and dendritic cells and these suppressive immunologic properties have led to considering its role in autoimmune and inflammatory diseases. In this way, the impact of VDR on experimental autoimmune prostatitis has been recently investigated using of VDR-Knockout mice. These knockout animals presented a more severe prostatitis with a higher lymphoproliferative response against prostate antigen and amplified levels of interferon $\gamma$ associated with an augmented of mononuclear cell infiltration. The authors concluded that VDR plays a role in pathophysiology of autoimmune prostatitis [28].

Pulmonary alveolar proteinosis (PAP) is an autoimmune disorder characterized by surfactant accumulation and neutralizing autoantibodies, granulocyte/macrophage colony stimulating factor, and deficiency of activin A (a cytokine implicated in regulating B-cell proliferation in alveolar macrophage). Recently studies also demonstrated deficiency in vitamin D target in bronchoalveolar lavage from these patients. A study investigated the effects of 1,25-dihydroxyvitamin $\mathrm{D}$ on activin of the macrophage alveolar from PAP and healthy subjects demonstrated that vitamin D increased activin A only in healthy controls but 
not in PAP patients. A deficient expression of vitamin D target genes (cathelicidin and thioredoxin interacting protein) was also observed in macrophages of PAP patients, suggesting that an impairment of activin expression may involve dysregulation of a novel species-specific vitamin D-activin A pathway in PAP [29].

Acknowledgments J.F. de Carvalho and R.M. Pereira were the recipients of grants from the Federico Foundation and CNPq (300665/ 2009-1 to JFC and 305691/2006-6 to RMRP).

Conflict of interest None.

\section{References}

1. Ascherio A, Munger KL (2007) Environmental risk factors for multiple sclerosis. Part I: the role of infection. Ann Neurol 61:288-299

2. Pender MP (2003) Infection of autoreactive B lymphocytes with $\mathrm{EBV}$, causing chronic autoimmune diseases. Trends Immunol 24:584-588

3. Pender MP (2009) Preventing and curing multiple sclerosis by controlling Epstein-Barr virus infection. Autoimmun Rev 8:563-568

4. Fraussen J, Vrolix K, Martinez-Martinez P, Losen M, De Baets MH, Stinissen P, Somers V (2009) B cell characterization and reactivity analysis in multiple sclerosis. Autoimmun Rev 8:654-658

5. Smolders J, Damoiseaux J, Menheere P, Cohen Tervaert JW, Hupperts R (2009) Association study on two vitamin D receptor gene polymorphisms and vitamin D metabolites in multiple sclerosis. Ann N Y Acad Sci 1173:515-520

6. Tajouri L, Ovcaric M, Curtain R, Johnson MP, Griffiths LR, Csurhes $\mathrm{P}$ et al (2005) Variation in the vitamin D receptor gene is associated with multiple sclerosis in an Australian population. J Neurogenet 19:25-38

7. Payne N, Siatskas C, Bernard CC (2008) The promise of stem cell and regenerative therapies for multiple sclerosis. J Autoimmun 31:288-294

8. Settin A, Ismail A, El-Magd MA, El-Baz R, Kazamel A (2009) Gene polymorphisms of TNF-alpha-308 (G/A), IL-10(-1082) (G/A), IL-6(-174) (G/C) and IL-1Ra (VNTR) in Egyptian cases with type 1 diabetes mellitus. Autoimmunity 42:50-55

9. Lempainen J, Vaarala O, Mäkelä M, Veijola R, Simell O, Knip M, Hermann R, Ilonen J (2009) Interplay between PTPN22 C1858T polymorphism and cow's milk formula exposure in type 1 diabetes. J Autoimmun 33:155-164

10. Iwase K, Shimada A, Kawai T, Okubo Y, Kanazawa Y, Irie J, Oikawa Y, Hirose H, Maruyama T, Itoh H (2009) FOXP3/Scurfin gene polymorphism is associated with adult onset type 1 diabetes in Japanese, especially in women and slowly progressive-type patients. Autoimmunity 42:159-167

11. Elagin RB, Balijepalli S, Diacovo MJ, Baekkeskov S, Jaume JC (2009) Homing of GAD65 specific autoimmunity and development of insulitis requires expression of both DQ8 and human GAD65 in transgenic mice. J Autoimmun 33:50-57

12. Kanazawa Y, Shimada A, Oikawa Y, Okubo Y, Tada A, Imai T, Miyazaki J, Itoh H (2009) Induction of anti-whole GAD65 reactivity in vivo results in disease suppression in type 1 diabetes. J Autoimmun 32:104-109
13. Khan MW, Sherwani S, Khan WA, Ali R (2009) Characterization of hydroxyl radical modified GAD65: a potential autoantigen in type 1 diabetes. Autoimmunity 42:150-158

14. Weenink SM, Lo J, Stephenson CR, McKinney PA, AnanievaJordanova R, Rees Smith B, Furmaniak J, Tremble JM, Bodansky HJ, Christie MR (2009) Autoantibodies and associated T-cell responses to determinants within the 831-860 region of the autoantigen IA-2 in Type 1 diabetes. J Autoimmun 33:147-154

15. Tsirogianni A, Pipi E, Soufleros K (2009) Specificity of islet cell autoantibodies and coexistence with other organ specific autoantibodies in type 1 diabetes mellitus. Autoimmun Rev 8:687-691

16. Zamani M, Spaepen M, Bex M, Bouillon R, Cassiman JJ (2000) Primary role of the HLA class II DRB $1 * 0301$ allele in Graves disease. Am J Med Genet 95:432-437

17. Jacobson EM, Huber A, Tomer Y (2008) The HLA gene complex in thyroid autoimmunity: from epidemiology to etiology. J Autoimmun 30:58-62

18. Tomer Y, Huber A (2009) The etiology of autoimmune thyroid disease: a story of genes and environment. J Autoimmun 32:231-239

19. Huber AK, Jacobson EM, Jazdzewski K, Concepcion ES, Tomer Y (2008) Interleukin (IL)-23 receptor is a major susceptibility gene for Graves' ophthalmopathy: the IL-23/T-helper 17 axis extends to thyroid autoimmunity. J Clin Endocrinol Metab 93:1077-1081

20. Ban Y, Tozaki T, Taniyama M, Nakano Y, Yoneyama K, Ban Y, Hirano $T$ (2009) Association studies of the IL-23R gene in autoimmune thyroid disease in the Japanese population. Autoimmunity 42:126-130

21. Parente Costa L, Bonfá E, Martinago CD, de Oliveira RM, Carvalho JF, Pereira RM (2009) Juvenile onset systemic lupus erythematosus thyroid dysfunction: a subgroup with mild disease? J Autoimmun 33:121-124

22. Somers K, Govarts C, Stinissen P, Somers V (2009) Multiplexing approaches for autoantibody profiling in multiple sclerosis. Autoimmun Rev 8:573-579

23. Tedesco A, D'Agostino D, Soriente I, Amato P, Piccoli R, Sabatini P (2009) A new strategy for the early diagnosis of rheumatoid arthritis: a combined approach. Autoimmun Rev $8: 233-237$

24. Aggarwal S, Gurney AL (2002) IL-17: prototype member of an emerging cytokine family. J Leuk Biol 71:1-8

25. Lan RY, Salunga TL, Tsuneyama K, Lian ZX, Yang GX, Hsu W, Moritoki Y, Ansari AA, Kemper C, Price J, Atkinson JP, Coppel RL, Gershwin ME (2009) Hepatic IL-17 responses in human and murine primary biliary cirrhosis. J Autoimmun 32:43-51

26. Yang K, Vega JL, Hadzipasic M, Schatzmann Peron JP, Zhu B, Carrier Y, Masli S, Rizzo LV, Weiner HL (2009) Deficiency of thrombospondin-1 reduces Th17 differentiation and attenuates experimental autoimmune encephalomyelitis. J Autoimmun 32:94-103

27. Castellanos-Rubio A, Santin I, Irastorza I, Castaño L, Carlos Vitoria J, RamonBilbao J (2009) TH17 (and TH1) signatures of intestinal biopsies of $\mathrm{CD}$ patients in response to gliadin. Autoimmunity 42:69-73

28. Motrich RD, van Etten E, Depovere J, Riera CM, Rivero VE, Mathieu C (2009) Impact of vitamin D receptor activity on experimental autoimmune prostatitis. J Autoimmun 32:140-148

29. Barna BP, Malur A, Dalrymple H, Karnekar R, Culver DA, Abraham S, Singh RJ, Brescia D, Kavuru MS, Thomassen MJ (2009) A novel 1,25-dihydroxyvitamin D-activin A pathway in human alveolar macrophages is dysfunctional in patients with pulmonary alveolar proteinosis (PAP). Autoimmunity 42:56-62 